\title{
Adenopatías generalizadas como presentación de la reacción leprótica tipo 2
}

\begin{abstract}
Gerzaín Rodríguez
Laboratorio de Patología, Instituto Nacional de Salud; Departamento de Patología, Facultad de Medicina, Universidad Nacional de Colombia, Bogotá, D.C., Colombia

Las reacciones en los pacientes con lepra son manifestaciones clínicas graves de inflamación aguda en las lesiones crónicas del enfermo, capaces de producir daño irreversible e incapacitante. Estudiamos un hombre de 46 años con reacción leprótica tipo 2, que consultó por fiebre, malestar general, sensación de obstrucción nasal, nódulos cutáneos y adenopatías generalizadas. El cuadro hemático mostró leucocitosis con neutrofilia. Entre varios diagnósticos clínicos sugeridos no se pensó en lepra. Una biopsia ganglionar demostró necrosis extensa del órgano que estaba infiltrado por polimorfonucleares y macrófagos espumosos, con necrosis de vénulas y depleción linfoide. No se hizo coloración de ZN, pero sí de Gomori, que tiñó muy bien los bacilos de Hansen, pero no se detectaron por el patólogo, que no hizo un diagnóstico concluyente. Veinte meses después, el paciente presentó síntomas semejantes con adenopatías generalizadas y nódulos cutáneos más numerosos, la biopsia de uno de los cuales demostró lepra lepromatosa con eritema nodoso leproso o reacción tipo 2. El tratamiento antileproso con poliquimioterapia y antirreaccional con talidomida curó al paciente, que 3 años después no presenta secuelas, pese a los 20 meses transcurridos para precisarse el diagnóstico. Comentamos este caso clínico y revisamos los factores predisponentes, la histopatología, los diagnósticos diferenciales de la adenopatía leprosa, la patogenia, el pronóstico y el tratamiento de la reacción tipo 2 en lepra, que constituye una urgencia médica, capaz de originar incapacidad grave y que, como en este enfermo, puede cursar con adenopatías como signos y síntomas predominantes.
\end{abstract}

Palabras clave: reacción leprótica, adenopatía leprosa, eritema nodoso leproso, reacción tipo 2.

\section{Generalized adenopathy as a manifestation of type 2 reactional leprosy}

Leprosy patient's reactions are severe clinical manifestations of acute inflammation of chronic lesions, capable of producing irreversible and invalidating damage. We studied a 46 year-old man with a type 2 leprosy reaction, who presented fever, cutaneous nodules, nasal obstruction and generalized adenopathy. The hemogram showed leucocytosis with neutrophilia. None of the initial diagnoses included leprosy. A lymph node biopsy revealed extensive necrotic areas infiltrated with polymorphonuclear lymphocytes, and foamy macrophages. Eosinophylic necrosis and thrombosis of venules with lymphoid nodule depletion was also in evidence. Ziehl Neelsen stain was not done, but the Gomori stain clearly showed Hansen's bacilli. These were were not detected by the pathologist and therefore a final diagnosis was not provided. Twenty months later, the patient presented similar symptoms, but with more generalized lymphadenopathy and presence of cutaneous nodules. Nodule biopsy showed lepromatous leprosy with erythema nodusum leprosum or type 2 reaction. Polychemotherapy treatment and anti-reaction treatment with thalidomide cured the patient. No sequelae were noted in 3 years following the treatment. A literature review of the type 2 reaction in leprosy is provided, including discussion of risk factors, histopathology, differential diagnosis for leprosy adenopathy, pathogenesis, prognosis, and treatment. Type 2 leprosy must be treated immediately upon diagnosis as it can cause serious and permanent tissue damage. As had occurred in the above patient, the disease can proceed with generalized and symptomatic lymphadenopathy.

Key words: leprosy pathology, leprosy lymphadenopathy, type 2 reaction, erythema nodosum leprosum 


\section{Caso clínico}

Hombre de 46 años, campesino, que consultó al hospital por presentar fiebre, malestar general, secreción nasal serosanguinolenta, sensación de obstrucción nasal, tos productiva con expectoración hialina, sudoración nocturna profusa, astenia, anorexia, pérdida de peso y "bolitas en la piel", junto con adenopatías cervicales, axilares e inguinales, dolorosas a la palpación, de una semana de evolución. Desde hacía tres días presentaba disuria. Había sido tratado con eritromicina y acetaminofén sin mejoría.

En el examen físico se encontró febril $\left(39,5^{\circ} \mathrm{C}\right)$, taquicárdico (98 pulsaciones/min), taquipneico (40 respiraciones/ $\mathrm{min}$ ) y con adenopatías dolorosas, cervicales, supraclaviculares, axilares e inguinales, bilaterales, móviles; tenía sensación de dolor en la región suprapúbica. Las orejas se describieron como infiltradas, duras, eritematosas y dolorosas a la palpación. Se observaron nódulos cutáneos en las costillas y en las axilas. Se hizo diagnóstico de síndrome febril prolongado e infección de vías urinarias bajas. Se sospechó tuberculosis, infección por VIH, enfermedad de Hodgkin, mononucleosis infecciosa y brucelosis.

No había antecedentes patológicos de importancia relacionados con la enfermedad actual.

Los exámenes paraclínicos mostraron: hemoglobina, $14 \mathrm{~g} / \mathrm{dl}$; hematocrito, $42 \%$; leucocitos, $10.500 / \mathrm{mm}^{3}$; neutrófilos, $80 \%$; linfocitos, $19 \%$; eosinófilos, $1 \%$; VSG, $17 \mathrm{~mm} / \mathrm{h}$. La baciloscopia de esputo fue negativa para bacilos ácido-alcohol resistentes; el parcial de orina mostró 6-9 leucocitos y 5-8 hematíes por campo de 40X; la glicemia y el ácido úrico fueron normales; el VDRL y los anticuerpos para HIV fueron negativos.

Se practicó biopsia de ganglios cervicales cuyo estudio de patología se informó así: macroscópicamente, el especimen era "un nódulo amarillento cauchoso, de $2 \mathrm{~cm}$ de diámetro mayor,

$\overline{\text { Correspondencia: }}$

G. Rodríguez, apartado aéreo 80334, Bogotá, D.C., Colombia Fax: 2220355 y 2220194

grodriguez@ins.gov.co

Recibido: 21/04/03; aceptado: 3/10/03 grisáceo y blando al corte"; microscópicamente se describió como: "ganglio linfático con abundantes granulomas en empalizada, con necrosis central, abundantes polimorfonucleares neutrófilos, cuerpos tingibles, tejido de granulación, histiocitos, con nódulos linfoides reactivos. Las coloraciones de PAS y Gomori no mostraron microorganismos. No se observa malignidad en el material analizado". Se informó como "linfadenopatía granulomatosa y necrotizante", con una nota que decía: "Debe descartarse TBC, fiebre por arañazo de gato, toxoplasmosis y leishmaniasis".

Con estos resultados, el médico tratante dedujo sin mayor fundamento que el paciente tenía tuberculosis e inició tratamiento estándar acortado supervisado con estreptomicina, pirazinamida, rifampicina e isoniacida, el cual sólo tomó durante 2 meses.

El paciente reingresó 20 meses después de la consulta relatada por presentar fiebre, malestar general, adenopatías cervicales, axilares e inguinales, múltiples, móviles, dolorosas, asociadas con lesiones de la piel, consistentes en placas eritematosas y algunos nódulos localizados en el abdomen, el tórax, el dorso de los antebrazos y los muslos (figura 1). Los exámenes paraclínicos mostraron: leucocitos, $19.900 \mathrm{~mm}^{3}$; neutrófilos, 92\%; linfocitos, 8\%; hemoglobina, $13 \mathrm{~g} / \mathrm{dl}$; hematocrito, $41 \%$; glucemia, $85 \mathrm{mg} / \mathrm{dl}$; urea, $34 \mathrm{mg} / \mathrm{dl}$; nitrógeno ureico, 15,8 $\mathrm{mg} / \mathrm{dl}$; creatinina, 0,4 mg/dl; proteínas totales, 6,1 $\mathrm{g} / \mathrm{dl}$; albúmina, 3,0 g/dl; globulinas, 3,1 g/dl; parcial de orina con albuminuria de $30 \mathrm{mg} / \mathrm{dl}$; factor reumatoideo negativo; VDRL, negativa, y radiografía de tórax normal. Con estos hallazgos se diagnosticó un eritema nudoso de etiología por determinar, una enfermedad del colágeno y vasculitis cutánea; se practicó biopsia de un nódulo del antebrazo que se fijó en formalina al $10 \%$ y se envió para estudio de patología.

La biopsia del nódulo cutáneo mostró extensos granulomas de macrófagos espumosos con abundantes polimorfonucleares y algunos linfocitos situados en la dermis y en la hipodermis (figuras 2 y 3 ); había necrosis fibrinoide de la pared de algunas de las vénulas hipodérmicas (figura 4); 
los nervios se veían engrosados, con infiltración discreta por los macrófagos espumosos (figura 5); la coloración de Fite-Faraco (1) mostró abundantes formas granulosas y ocasionales bacilos enteros ácido-alcohol resistentes en todos los infiltrados y en los nervios (figura 6); la imagen se interpretó como lepra lepromatosa en reacción tipo 2 (R-2 o eritema nodoso leproso, ENL).

Con estos hallazgos en la piel, se solicitaron los cortes y bloques de parafina del ganglio linfático cuya biopsia se había obtenido 20 meses antes, en los cuales se observó que la masa de $2 \mathrm{~cm}$ descrita macroscópicamente correspondía a conglomerados de ganglios linfáticos en los cuales el tejido linfoide estaba disminuido y predominaban extensos acúmulos corticales y medulares de polimorfonucleares, entre los cuales se apreciaban grandes cantidades de macrófagos espumosos, llenos de un material granular o fibrilar finamente basófilo (figuras 7 a 9); con la coloración de plata metenamina (Gomori), la cual se había realizado en el estudio inicial del ganglio, se observaron abundantes bacilos teñidos de negro, situados dentro de estos macrófagos vacuolados (figura 10); con la coloración de Fite-Faraco, que no se había realizado en el estudio previo, se observaron formas granulares y abundantes bacilos ácido-alcohol resistentes enteros dentro de los macrófagos (figura 11). Había, además, necrosis fibrinoide de la pared de algunas vénulas y la inflamación se extendía a la grasa periganglionar. Varios filetes nerviosos periganglionares no se encontraban afectados por el proceso y no contenían bacilos en la coloración de Fitte-Faraco. Con estos hallazgos, se concluyó que el ganglio linfático tenía lepra lepromatosa con signos de reacción leprótica tipo dos.

\section{Evolución del paciente}

El paciente se sometió a terapia multidroga (TMD) antileprosa y a tratamiento de la reacción 2 con talidomida, con mejoría importante de su cuadro clínico. Un examen clínico exhaustivo practicado 22 meses después del inicio de su enfermedad demostró discreta disminución de la sensibilidad en lesiones glúteas eritematoescamosas y en el dorso de los antebrazos; no se encontró engrosamiento ni dolor a la palpación de los troncos nerviosos y no había pérdida de la fuerza muscular de manos o pies, ni alteraciones oculares. En septiembre de 2003, el paciente estaba curado de la lepra, sin secuelas y no había vuelto a presentar episodios reaccionales.

\section{Discusión}

\section{Definición de las reacciones}

Las reacciones en lepra son respuestas inmunológicas de hipersensibilidad, que se manifiestan con signos y síntomas de inflamación aguda en los sitios afectados por la enfermedad (1); el sistema inmune trata de destruir el bacilo de Hansen produciendo interleucinas de la respuesta $\mathrm{TH}-1$, lo cual también es nocivo para el huésped (véase patogenia). Se clasifican en tipo 1 o de reversión, tipo 2 o de eritema nodoso leproso $(2,3)$, que es la diagnosticada en el paciente presentado y tipo 3 o fenómeno de Lucio (1).

La reacción 2 se presenta abruptamente en episodios que duran 3 a 12 semanas, especialmente las lesiones cutáneas, que desaparecen espontáneamente. Algunos pacientes pueden tener un solo episodio, pero la recurrencia, la persistencia y la cronicidad de la reacción son comunes. En este paciente, es posible que los nódulos cutáneos, que duran 3 a 5 días, ya no estuvieran presentes cuando se le examinó la primera vez, o que se confundieran con adenopatías.

Las principales características clínicas de la reacción 2 son: 1) fiebre con síntomas y signos de malestar general, usualmente muy importantes, que obligan a consultar al enfermo; 2) neuritis, cuya magnitud define la gravedad principal del cuadro clínico; puede generar incapacidad grave y cataloga a la reacción como una urgencia (1-5); 3) conjuntivitis, iridociclitis y, en general, "ojo rojo", que también constituyen una urgencia médica; 4) nódulos cutáneos y placas eritematosas, frecuentes, pero no siempre presentes, que hacen llamar "eritema nodoso leproso (ENL)" a la reacción 2, nombre muy usado pero inadecuado, porque sólo enfatiza una manifestación del cuadro clínico; por eso, Jopling propuso denominarla reacción $2(2,3)$; 5) edema doloroso e incapacitante de las manos y de los pies $(2) ; 6)$ epistaxis, obstrucción y secreción 


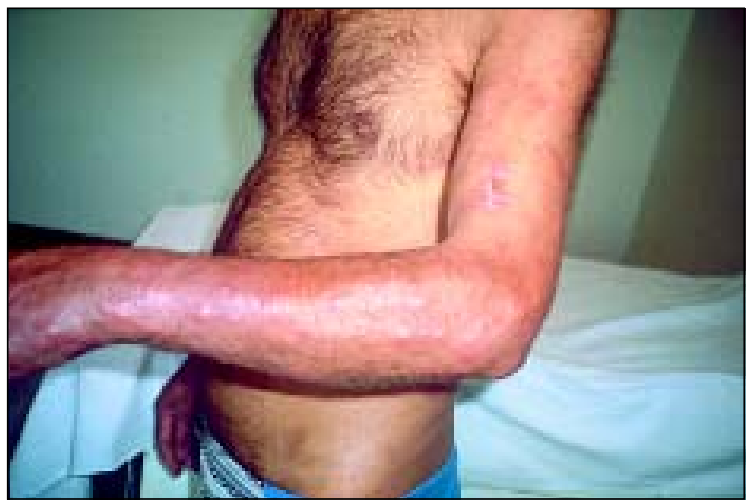

Figura 1. Numerosos nódulos del dorso del antebrazo, de la cara externa del brazo y del tronco, sobre un fondo eritematoso.
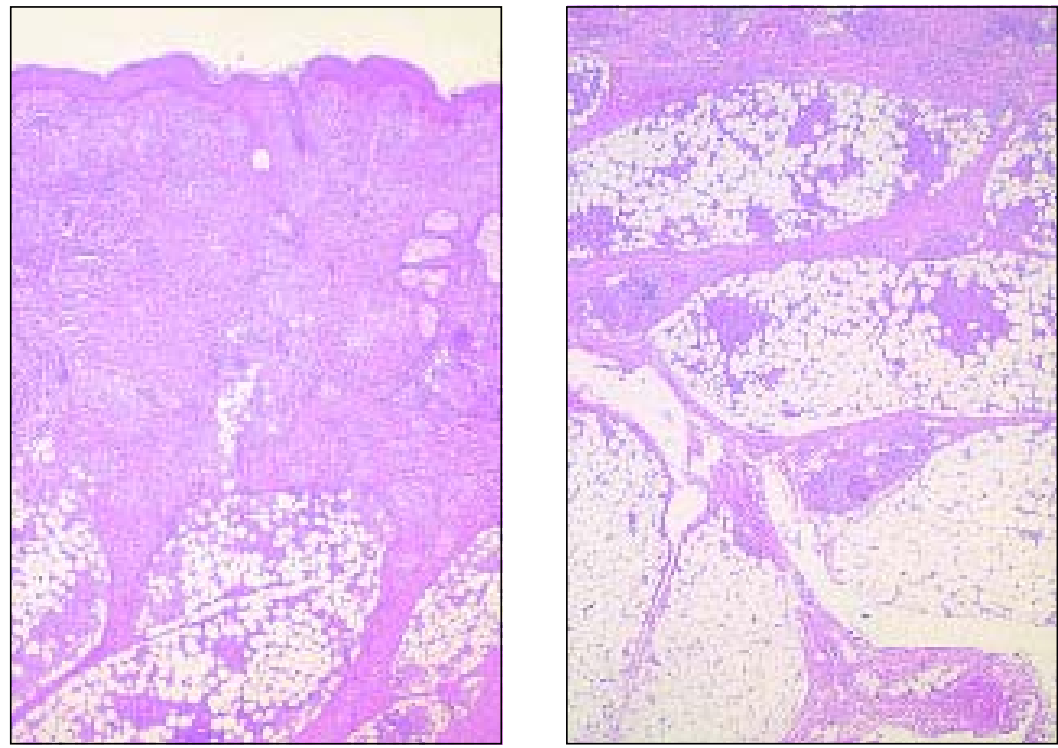

Figuras 2 y 3. Biopsia de un nódulo cutáneo. Imágenes panorámicas. Infiltrados de células claras en la dermis y dentro de los lobulillos adiposos. En el tabique interlobulillar inferior de la figura 3 se observa un nervio engrosado, que se amplía en la figura 5 . HE, 2,5X.

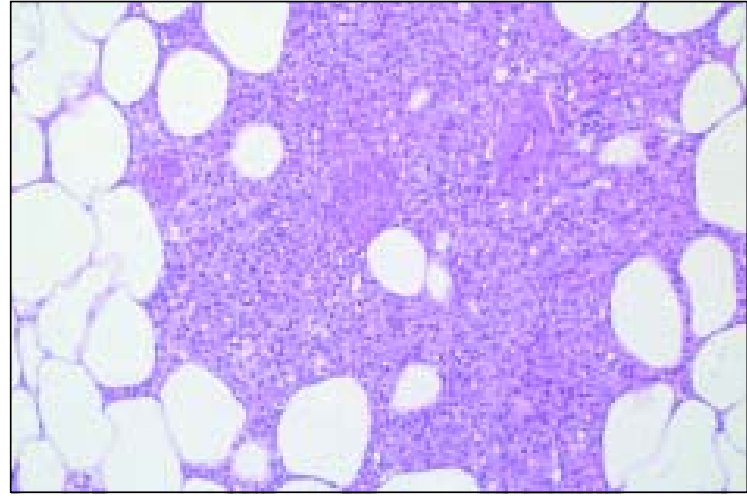

Figura 4. Inflamación intralobulillar hipodérmica con macrófagos espumosos, abundantes polimorfonucleares y trombosis con necrosis fibrinoide de vasos pequeños. La imagen es típica de ENL. HE, 20X.

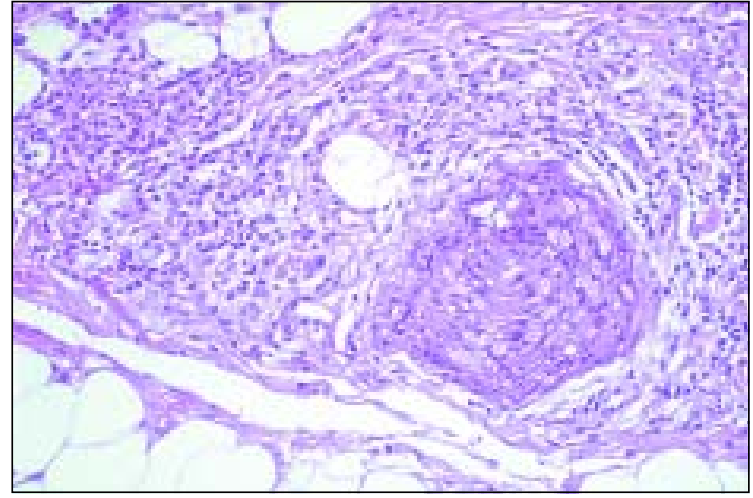

Figura 5. Nervio subcutáneo visto en la figura 3. Presenta engrosamiento perineural leve y macrófagos espumosos en su interior. Está rodeado por infiltrado linfoplasmocitario rico en células de Virchow. Tanto en este infiltrado como dentro del nervio, se demostraron formas granulosas ácidoalcohol resistentes. HE, 25X. 


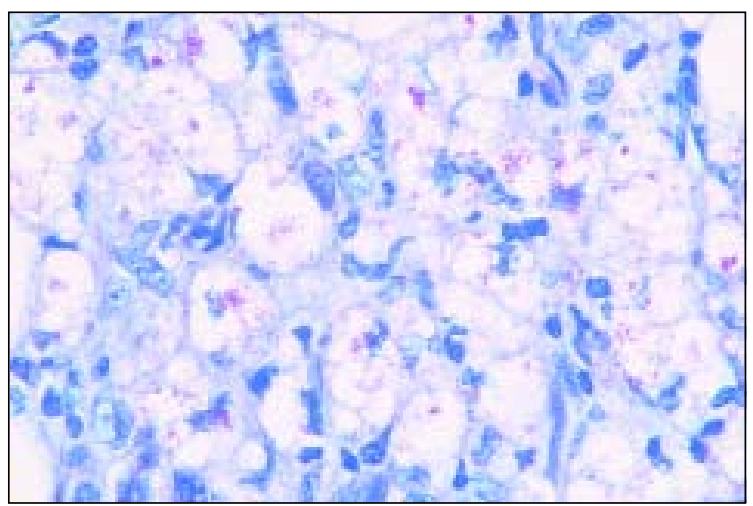

Figura 6. Abundantes formas granulosas dentro de los macrófagos espumosos con pocos bacilos enteros; discreto infiltrado de polimorfonucleares. FiteFaraco, 80X.

Figuras 7 a 11. Imágenes del ganglio linfático.

Figura 7. Escasos nódulos linfoides. Amplio infiltrado de células claras y borramiento de la cápsula.

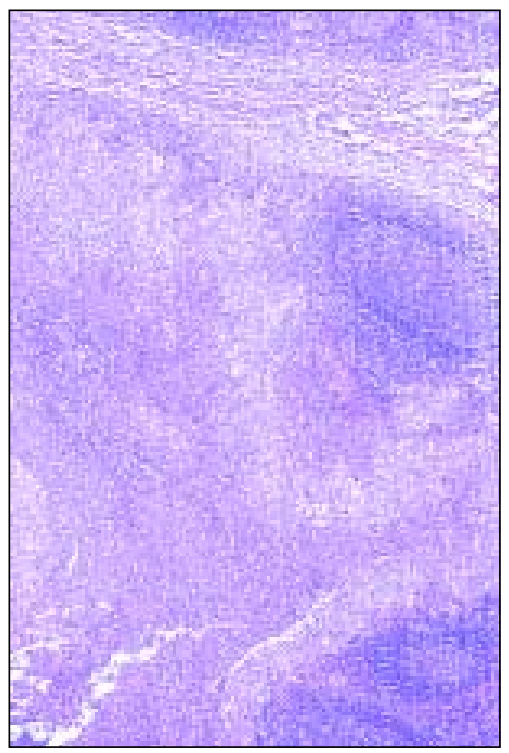

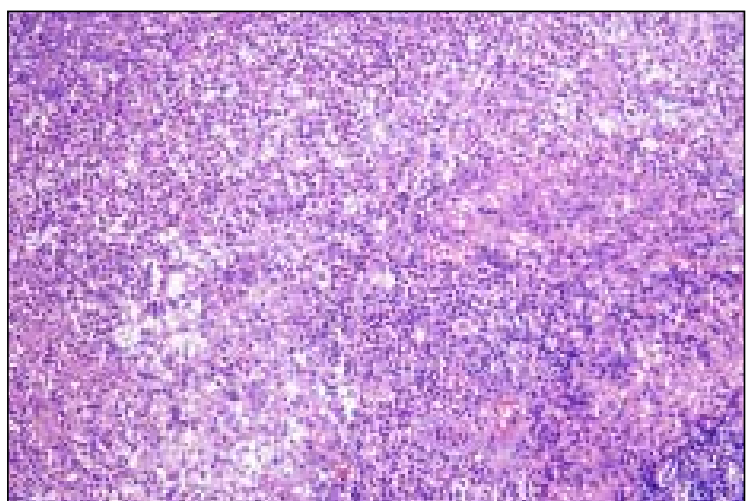

Figura 8. A mayor aumento, en el infiltrado inflamatorio se ven abundantes células vacuoladas y polimorfonucleares con pocos linfocitos. HE, 20X.

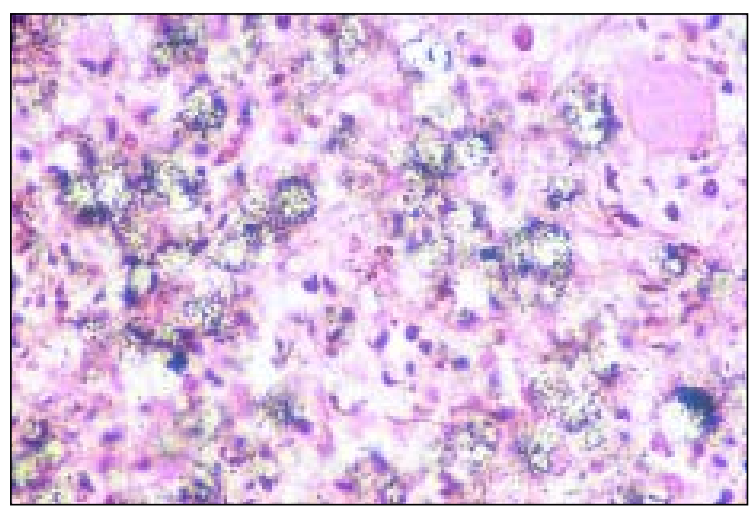

Figura 10. La coloración de plata metenamina muestra abundantes bacilos y formas granulosas teñidas de negro, en los macrófagos vacuolados. Gomori, 80X.

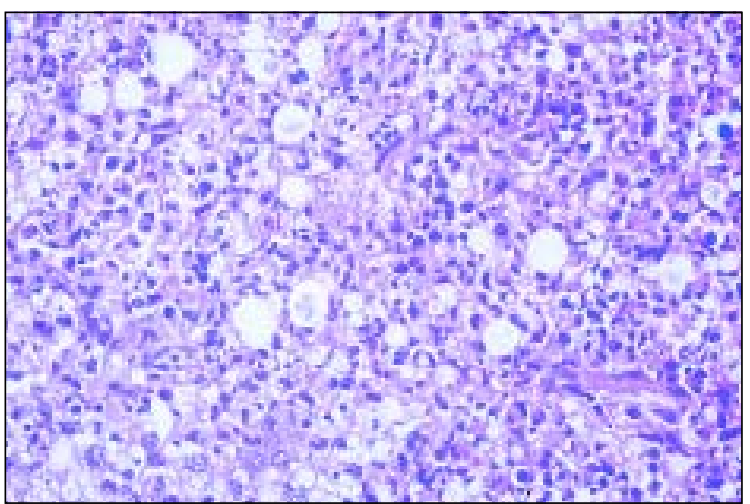

Figura 9. Las células espumosas o vacuoladas contienen material particulado basófilo. Se ven numerosos polimorfonucleares, algunos con leucocitoclasia. La imagen es típica de lepra lepromatosa en reacción dos. HE, 50X.

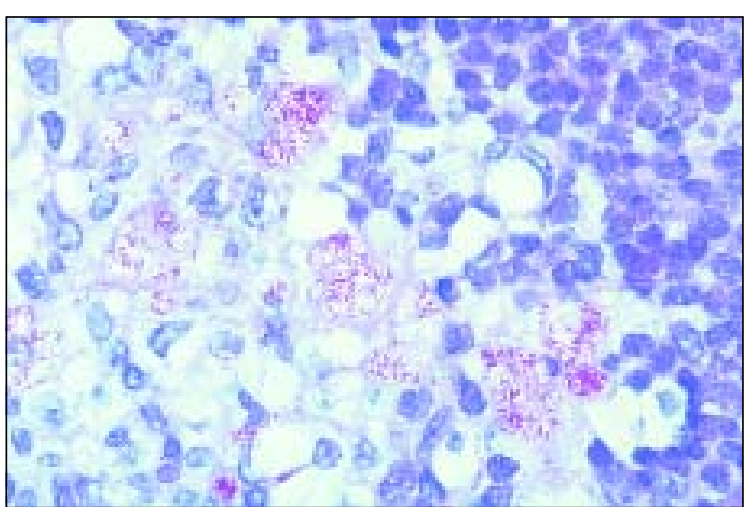

Figura 11. La coloración muestra abundantes formas granulosas, algunos bacilos enteros y globias en las mismas células del ganglio linfático. Fite-Faraco, 100X. 
nasal mucosanguinolenta $(6,7) ; 7)$ orquitis y epididimitis, a veces muy severas e incapacitantes; 8 ) artritis de grandes y pequeñas articulaciones $(8,9)$; 9) glomerulonefritis por complejos inmunes $(10)$; 10) adenopatías dolorosas, generalizadas $(6,11,12)$, que dominaron el cuadro clínico de nuestro enfermo.

\section{Análisis del caso clínico}

Se trata de un paciente con enfermedad febril aguda, con notorio malestar general, síntomas respiratorios superiores y adenopatías generalizadas. Éstas dominaron el cuadro clínico e hicieron pasar desapercibidas las "bolitas en la piel" y los "nódulos de las costillas", así como las lesiones de las orejas, claves todas, que hubieran llevado al diagnóstico de lepra en reacción tipo 2, con componente importante de eritema nodoso leproso, fácilmente confirmable con la baciloscopia para lepra y con la biopsia de piel.

Los síntomas respiratorios también fueron notorios y algunos de ellos forman parte de la reacción tipo 2 en el enfermo, reacción inflamatoria que afecta varios de los sitios en donde ocurre la lepra lepromatosa, como la nariz (1), órgano en el que puede comenzar esta reacción con secreción mucopurulenta, sensación de obstrucción y epistaxis $(1,6,7)$, como ocurrió en nuestro paciente. La sensación de obstrucción nasal y el edema de los miembros inferiores son síntomas y signos precoces de la lepra lepromatosa (2). El mal estado general es una característica esencial del paciente con reacción tipo 2 , junto con la fiebre y los dolores generalizados, articulares o no, que inducen a consultar al enfermo. La disuria no guarda relación con la reacción 2 .

Otro aspecto importante para resaltar es la presentación inicial de la lepra con la reacción 20 de ENL, como causa de la consulta, lo cual ocurre entre nosotros en el $20 \%$ de los pacientes con este síndrome (1) y hasta en el $69 \%$ de los casos nuevos de otros estudios (13). La reacción 2 puede ocurrir como primera manifestación de la lepra, durante su tratamiento o luego de terminado éste. (2-5). Se ha visto que $9 \%$ de los pacientes que terminan su poliquimioterapia (PQT) antileprosa pueden presentar eritema nodoso leproso (14).
No sabemos cuántos episodios reaccionales presentó el enfermo durante los 20 meses de evolución de su enfermedad; es poco probable que hubiera sido sólo uno.

Las adenopatías agudas, generalizadas, junto con el cuadro febril y el mal estado general sugieren un proceso infeccioso, apoyado por el cuadro hemático, que mostró leucocitosis con neutrofilia. Algunas enfermedades infecciosas con adenopatías se incluyen en el cuadro 1.

\section{La adenopatía en lepra}

El ganglio linfático se compromete en todas las formas de lepra, inclusive en la lepra indeterminada (15), aunque otros autores niegan el compromiso ganglionar en esta forma de la enfermedad (16); los ganglios afectados son los del área que drena las lesiones cutáneas, formando una adenopatía regional; rara vez es generalizada en la lepra lepromatosa o en la lepra dimorfa lepromatosa. Las adenopatías son preferencialmente inguinales, cervicales, axilares, epitrocleares y preauriculares $(15,17)$; sólo en la lepra lepromatosa alcanzan más de $1 \mathrm{~cm}$ o son más sintomáticas (15). En enfermos de lepra del Sanatorio de Contratación (Santander) se describían con tamaños "desde una lenteja hasta el de un huevo de gallina o una naranja" (17). En la reacción 2 son características, generalizadas, importantes, dolorosas y pueden dominar el cuadro clínico e, inclusive, ser su forma de presentación, sin lesiones cutáneas aparentes $(6,11)$, o con lesiones cutáneas poco manifiestas, como en la consulta inicial de nuestro paciente, lo cual no justifica que estas lesiones hayan pasado desapercibidas. En el transcurso del tiempo, sin tratamiento adecuado, aparecerán

Cuadro 1. Adenopatías en enfermedades infecciosas.
1. Sífilis secundaria
2. Lepra lepromatosa; reacción 2
3. Enfermedad por arañazo de gato
4. Endocarditis bacteriana
5. Linfogranuloma venéreo
6. Toxoplasmosis
7. Leishmaniasis
8. Histoplasmosis, paracoccidiodomicosis
9. Sida
10. Mononucleosis infecciosa
11. Enfermedad de inclusión citomegálica 
placas y nódulos cutáneos, pequeños, profundos, sobre la piel aparentemente sana de los antebrazos, la cara, los muslos y el tronco, en episodios, con involución y aparición de nuevos nódulos en cualquier parte de la piel, excepto en el cuero cabelludo (1-5). Las adenopatías cervicales dolorosas y la neuritis local concomitante pueden originar rigidez nucal que, junto con el cuadro séptico general, conducen a sugerir el diagnóstico de meningitis (6-7). El ganglio linfático y los nervios son los principales órganos en los cuales persisten bacilos viables una vez terminada la poliquimioterapia antileprosa de 2 años de duración (18).

\section{Laboratorio}

En la R-2 es característica la leucocitosis con neutrofilia (11), que llegó en el enfermo a 19.900 leucocitos $/ \mathrm{mm}^{3}$, con $92 \%$ de polimorfonucleares. Hay aumento de la VSG, anemia discreta y albuminuria; ésta refleja el depósito de complejos inmunes en el glomérulo (10); no estaba presente en la consulta inicial del paciente pero fue evidente 20 meses después de comenzada la enfermedad. El examen parcial de orina es obligatorio en todo paciente con lepra y, especialmente, en las reacciones porque informa sobre el estado del riñón (10).

También puede haber serología positiva falsa para sífilis (1), hecho que no estaba presente en el enfermo y que probablemente requiera años con la reacción para producirse.

La hipergammaglobulinemia y el aumento de la IgM pueden ser discretos, de acuerdo con la cronicidad del proceso. El complemento ha sido normal en los pocos casos en los que hemos registrado este estudio, pese a que se consume en la reacción antígeno-anticuerpo que ocurre en la reacción 2. Es común que esté aumentado por las fracciones C3a y C3d $(5,19)$; esta última es la que se fija a la reacción antígeno-anticuerpo para formar los complejos inmunes, locales o circulantes.

También pueden ser falsos positivos el factor reumatoide y los anticuerpos antinucleares. La proteína sérica $A$, precursora del amiloide, está elevada en el $100 \%$ de los casos y predice la persistencia de la reacción (20). No es una técnica usual de laboratorio. La R-2 crónica, persistente, es una causa de amiloidosis secundaria sistémica $(1,10)$. También está elevada la proteína $\mathrm{C}$ reactiva en esta reacción (20).

\section{Patología}

La reacción 2 en la piel predominantemente es una hipodermitis neutrofílica, instalada sobre un fondo de macrófagos espumosos que indican el estado lepromatoso o dimorfo lepromatoso; en cerca de la mitad de los casos hay vasculitis necrotizante de vénulas y pequeñas venas; en los casos más graves se ven edema y abscesos en la dermis (1). El infiltrado notorio de linfocitos 0 plasmocitos es excepcional. La coloración de FiteFaraco demuestra abundantes formas granulosas con pocos o ningún bacilo entero; los nervios muestran edema y penetración de los polimorfonucleares al endonervio. Ocasionalmente puede haber eritema nodoso leproso sin polimorfonucleares (20) y en el eritema nodoso leproso crónico pueden predominar los linfocitos en la hipodermis.

Los cambios histopatológicos de la reacción 2 en el ganglio linfático son característicos y equivalentes a los de la piel $(11,12)$ : acúmulos de polimorfonucleares, a veces muy extensos, que forman amplios abscesos que reemplazan el tejido linfoide, mezclados con gran número de macrófagos vacuolados o espumosos y presencia de vasculitis de vénulas y de otros vasos pequeños, evidenciada por trombosis y necrosis fibrinoide de su pared que está comprometida por el infiltrado y rodeada por él. En la biopsia ganglionar del paciente, inexplicablemente no se practicó la coloración de Ziehl-Neelsen o de FiteFaraco, que hubieran revelado abundantes bacilos y formas granulosas en los macrófagos, como lo demostramos después. Un hallazgo especial fue la presencia de gran cantidad de bacilos fagocitados, en la coloración de plata metenamina (Gomori). Este hecho es útil de saber porque esta coloración demuestra muchas bacterias, como el bacilo de Hansen (6,21), Afipia felis, Mycobacterium avium, Klebsiella y muchas más. Los patólogos deben tener en cuenta este hecho porque es común solicitar la coloración de plata metenamina, PAS y ZN en el estudio de procesos inflamatorios supurativos y granulomatosos del 
ganglio linfático. Es importante resaltar que el bacilo de Hansen es menos ácido-alcohol resistente que el bacilo de Koch y que las técnicas para teñirlos no son equivalentes $(1,22)$. Para demostrar el bacilo de Hansen se hace una técnica que demuestra su ácido-resistencia al $\mathrm{H}_{2} \mathrm{SO}_{4}$ al $5 \%$; es esencial hacer la coloración con el control adecuado, que debe ser un caso de lepra y no uno de tuberculosis $(1,22)$.

\section{Patogenia}

El eritema nodoso leproso representa una reacción de hipersensibilidad del huésped, que cambia su respuesta inmune ante antígenos o epítopos definidos de proteínas del bacilo de Hansen (23). Ese cambio inmunológico desencadena una inflamación aguda que trata de destruir el bacilo pero que también es gravemente nociva para el huésped. Los mecanismos que intervienen involucran la inmunidad celular y la humoral, mediadas a través de numerosas interleucinas. Inicialmente hay un aumento de los linfocitos T4 ayudadores, hasta 100 veces mayor que los vistos en la lepra lepromatosa y que alcanzan una densidad apenas menor que la presente en la lepra tuberculoide $(24,25)$; diversas células sanguíneas y cutáneas producen IFN $\gamma$, FNT $\alpha$, IL-1, IL-2, IL-6, IL-8, FNTB, IL-12 (26-29), curiosamente, las mismas interleucinas que están aumentadas en la reacción 1 o de reversión (26). La IL-4 y la IL-10 se expresan en menor cantidad y la IL-10 puede demostrarse en los linfocitos de la sangre periférica pero no en las lesiones $(26,27)$. Todas estas interleucinas son las responsables de la fiebre y el mal estado general, principalmente el factor de necrosis tumoral alfa; los anti-inflamatorios bloquean su expresión y alivian al enfermo (30). Los metabolitos del óxido nítrico están aumentados en la orina (31), lo que refleja la activación de macrófagos, que se tornan capaces de destruir los bacilos. Los niveles de IL-4 permanecen bajos, aunque sólo en 3 pacientes con reacción 2 persistente se mantuvieron elevados (30).

En resumen, la $\mathrm{R}-2$ es un proceso inflamatorio agudo antibacilar pero deletéreo para el huésped en el cual se cambia la respuesta inmune del tipo TH2 al TH1, cambio insuficiente para recuperar la respuesta adecuada contra el bacilo, para ascender en el espectro inmunológico o para hacer positiva la lepromina (32); no obstante, las formas y bacilos granulosos presentes en el sitio de la reacción no son viables (33). La R-2 induce la formación de complejos inmunes locales y circulantes (34-36) que amplifican a distancia el daño celular y tisular ya producido por el exceso de síntesis de interleucinas.

\section{Factores desencadenantes y factores de riesgo}

El cuadro 2 muestra las condiciones o circunstancias que favorecen la aparición de la R-2. Es posible que los cambios inmunológicos que algunos de ellos tienen, influyan en la reacción aguda del paciente.

Los factores de riesgo son:

1. Tener lepra lepromatosa (LL) y dimorfa lepromatosa (DL). La R-2 es más frecuente en pacientes LL que en los $\operatorname{DL}(2,14,17,37)$.

2. Índice bacilar alto (14), de 2-3, en nuestro Programa.

3. Infiltración de las lesiones cutáneas (14), es decir, que son firmes, duras, con mayor extensión a la palpación que la sugerida por la sola inspección.

4. La PQT podría tener algún efecto al liberar antígenos bacilares desencadenantes de la reacción, hecho más aparente en los enfermos con LL (19). La R-2 es más frecuente después de 6 meses de iniciado el tratamiento y anteriormente, con la monoterapia con DDS, era más común luego de 2-3 años de tratamiento (2). Este factor ha cambiado con la introducción de clofazimine en la PQT. De hecho, el clofazimine es una droga importante en el tratamiento de la reacción 2 por su poder

Cuadro 2. Factores de riesgo.

- LL o LDL con infiltración e índice bacilar 2 - 3

- Infección o enfermedad intercurrente, por ejemplo, malaria, varicela, tiñas.

- Cirugías

- Estrés

- Vacunas: viruela, BCG

- Embarazo y puerperio

- Ingestión de KI

- Tratamiento antileproso 
antiinflamatorio $(5,38)$. Si se usa en la PQT, la frecuencia de reacción 2 disminuye considerablemente, de cerca del $30 \%-50 \%$ en la LL a cerca del $5 \%$ (38); en los pocos casos en los que no se ha usado para el tratamiento de la $L L$, la frecuencia de las reacciones permanece igual (38). Recordemos que la R-2 puede ser la causa de consulta en un alto porcentaje de pacientes, que no han recibido tratamiento antileproso. Este no debe suspenderse durante la reacción.

5. Suspensión de la terapia PQT cuando el paciente tiene todavía baciloscopia positiva. Esta condición ocurre en pacientes multibacilares en los que luego de dos años de tratamiento se suspende la PQT, según las normas del programa de control de la lepra y que tienen un índice bacilar alto, mayor o igual a 4 en la escala de Ridley (o de 2-3 en la muestra). Un factor que propicia esta reacción post-PQT es la suspensión del clofazimine (39).

6. Edad y sexo: el ENL es más frecuente en hombres entre 20 y 40 años de edad. Los pacientes $L L$ mayores de 40 años tienen riesgo significativamente menor de hacer LL $(14,37)$. La reacción 2 ocurre también en los niños (1).

7. El embarazo y el puerperio son frecuentes ocasiones en las que ocurre la reacción 2 $(1,40)$. Es posible que en el puerperio, la reacción 2 cambie a reacción 1 , al recuperar la paciente una mejor respuesta inmune $(1,40)$. La reacción 2 ocurre con mayor frecuencia en el tercer trimestre del embarazo y en los 6 primeros meses de lactancia $(1,40)$.

\section{Diagnóstico diferencial de la adenopatía leprosa}

Frente a un paciente con malestar general importante, enfermedad aguda, fiebre y adenopatías, se deben considerar numerosas entidades infecciosas multisistémicas (cuadro 1). La presencia de adenopatías debe estudiarse de acuerdo con su localización, su número, el tiempo de evolución, la edad del paciente y otros factores epidemiológicos, pues estos datos indican si puede ser una infección bacteriana, venérea, viral, o una metástasis (41). Por otra parte, una adenopatía localizada es de enfoque muy diferente a las adenopatías generalizadas que por definición involucran 2 o más conglomerados ganglionares.

La tuberculosis pulmonar es una causa de eritema nodoso, hipodermitis que no se identifica microscópicamente como de origen específico por TBC; no difiere de cualquier otro EN, como sí lo hace el ENL, el único que demuestra su origen específico en la biopsia. La tuberculosis ganglionar ocurre en niños y personas jóvenes, con adenopatías en el cuello, las axilas y las ingles, que pueden drenarse por trayectos fistulosos a la piel, constituyendo la variedad cutánea de la tuberculosis denominada escrofuloderma. En esta entidad, tanto la piel como el ganglio linfático muestran inflamación granulomatosa tuberculoide, con amplia necrosis de caseificación rodeada de células epitelioides y gigantes multinucleadas. En la coloración de ZN es difícil demostrar los bacilos en nuestra experiencia, pero se pueden y se deben demostrar por el cultivo o por PCR. El cultivo, además, tiene la ventaja de detectar su origen eventual por micobacterias atípicas. La tuberculosis y la lepra lepromatosa pueden presentarse simultáneamente en el mismo ganglio linfático (42).

La infección ganglionar por Mycobacterium avium es rara; afecta a pacientes inmunosuprimidos, especialmente por sida. Compromete principalmente el pulmón desde donde se disemina a otros órganos, entre éstos los ganglios linfáticos, en los cuales se presenta una reacción granulomatosa con macrófagos espumosos, repletos de bacilos ácido-alcohol resistentes, sin necrosis ni formación de células multinucleadas. Pueden sugerir lepra pero no hay afección neural clínica ni histológica. El diagnóstico definitivo es por cultivo (16).

La sífilis secundaria produce una erupción cutánea de máculas, pápulas y maculopápulas anulares y descamativas, generalizadas, con compromiso de las palmas y de las plantas. También ocasiona úlceras de la mucosas, especialmente de la boca y vegetaciones anogenitales prominentes, llamados condilomas lata o planos. No ocasiona nódulos subcutáneos. Las adenopatías son comunes, generalizadas, 
pequeñas, menores de $2 \mathrm{~cm}$, no confluentes, ni dolorosas, móviles, que al microscopio muestran hiperplasia notoria de los centros germinales, prominencia reticulohistio-citaria y sinusoidal, y abundancia de plasmocitos que rodean los vasos corticales y medulares. A medida que avanza la enfermedad se ven granulomas epitelioides con células gigantes de Langhans, sin necrosis (43). Todas las lesiones de la sífilis secundaria son muy ricas en espiroquetas que se pueden demostrar con la coloración de Warthin-Starry. La serología (VDRL) es positiva, examen que también puede ser positivo en pacientes con ENL persistente. Nuestro paciente no presentó serología falsa positiva para lúes.

En la brucelosis hay hiperplasia de los centros germinales e inflamación aguda con presencia de polimorfonucleares en el ganglio. No tenemos experiencia con esta entidad, cuyo diagnóstico principal se hace por pruebas inmunológicas del laboratorio clínico.

La enfermedad por arañazo de gato predomina en menores de 20 años, en los que se presentan el $80 \%$ de los casos $(44,45)$. Da origen a una adenopatía única, voluminosa, hasta de $10 \mathrm{~cm}$ de diámetro, localizada en la región proximal al arañazo; responde poco al tratamiento y puede desaparecer espontáneamente en pocos meses. Histológicamente se caracteriza por abscesos estelares de polimorfonucleares, amplia necrosis de caseificación y por un granuloma periférico a estas áreas, constituido por células epitelioides, células gigantes, linfocitos y plasmocitos (45). También puede haber zonas con proliferación vascular con acúmulos de neutrófilos y de masas eosinófilas que corresponden a las colonias bacterianas, en un patrón inflamatorio semejante al de la angiomatosis bacilar de la piel $(44,45)$. Las bacterias causantes, Afipia felis o Bartonella henselae, se pueden demostrar con las coloraciones que usan sales de plata (45).

En las leishmaniasis se presenta compromiso del ganglio linfático en todas las formas de la enfermedad (46). En la leishmaniasis visceral se pueden palpar adenopatías móviles, pequeñas, de $1 \mathrm{~cm}$ de diámetro o menos, en las ingles y en el cuello, como parte de un cuadro clínico en el que predominan la hepatomegalia y la esplenomegalia. En Colombia, la enfermedad afecta principalmente a menores de 5 años, de áreas endémicas precisas (46). En la leishmaniasis cutánea se comprometen los ganglios que drenan la úlcera. La adenopatía varía en tamaño de 2 a $10 \mathrm{~cm}$ de diámetro y se demuestra hasta en el $77 \%$ de los enfermos (47). Puede ser la primera y la única manifestación de la leishmaniasis (48), ser concomitante con la úlcera, o persistir después de curada ésta.

Las biopsias ganglionares de leishmaniasis muestran patrones inflamatorios diversos que reflejan la respuesta inmune del huésped. En la leishmaniasis visceral se ven abundantes amastigotes dentro de los macrófagos vacuolados. En las adenopatías de las formas mucocutáneas o mucosas hay granulomas de macrófagos espumosos o epitelioides, con variable cantidad de amastigotes fagocitados, a veces muy numerosos (46). La biopsia no permite decir qué tipo de leishmaniasis tiene el paciente. La inmunohistoquímica ayuda a demostrar los amastigotes (46).

La toxoplasmosis puede desencadenar un cuadro febril con compromiso sistémico y muy mal estado general pero las adenopatías son de localización cervical y no hay manifestaciones cutáneas; histológicamente, el ganglio tiene histiocitos epitelioides que forman numerosos y diminutos granulomas (49); no se ven granulomas macrofágicos como en la lepra, la localización es de preferencia en las zonas cortical y paracortical, acompañada de hiperplasia folicular; en algunos casos se demuestra la forma quística del parásito (50), pero siempre se deben realizar estudios serológicos y cultivos.

La histoplasmosis tiene un espectro de presentación en el ganglio linfático según el estado inmune del huésped que va desde granulomas tuberculoides o epitelioides con pocos hongos, difíciles de demostrar con las coloraciones especiales, hasta la caracterizada por necrosis de coagulación extensa o por reemplazo del ganglio por macrófagos espumosos que albergan enorme número de histoplasmas. Clínicamente también hay un espectro de la enfermedad, que 
cursa con hepatoesplenomegalia, leucopenia, plaquetopenia y anemia (51).

La adenopatía en la infección por HIV es una fase importante de la enfermedad, que también es generalizada y persistente. Su aspecto histológico varía según la progresión de la enfermedad. Inicialmente hay hiperplasia folicular notoria con centros germinales prominentes y aspecto irregular, bizarro de los folículos, que conservan la zona marginal; no se ven granulomas; con el tiempo, los folículos son invadidos por linfocitos pequeños que deprimen y distorsionan el centro germinal, que aparece pequeño, lisado; es común ver células gigantes multinucleadas que representan fusión linfocitaria inducida por el virus. A medida que la adenopatía progresa el tejido linfoide y los nódulos son menos aparentes, hay aumento del número de vasos, fibrosis y notoria depleción linfoide, signo de la progresión hacia el sida (52).

En resumen, la adenopatía por enfermedad infecciosa puede diagnosticarse con certeza razonable en la biopsia ganglionar, en la mayoría de los casos.

\section{Pronóstico}

La reacción 2 es una urgencia médica, especialmente cuando afecta los troncos nerviosos y los ojos y cuando compromete de manera grave el estado general del enfermo (53); puede requerir hospitalización y el manejo no sólo quimioterápico, sino fisioterápico para prevenir el daño neural. El edema de las manos y de los pies, con el cual puede comenzar la R-2, es capaz de causar deformación o incapacidad grave si no se maneja vigorosamente con antiinflamatorios, inmovilización y fisioterapia temprana (54). La glomerulonefritis crónica por complejos inmunes regresa con la supresión de la reacción (10). El daño neural troncular conduce a parálisis del orbicular de los párpados, de las manos y de los músculos del pie. La orquiepididimitis en ocasiones es muy grave; el daño testicular que ocasiona conduce a azoospermia, esterilidad y ginecomastia, tal como sucede en la lepra lepromatosa (55). La R-2 persistente fue una causa importante de amiloidosis secundaria sistémica $(1,2,10)$. Antes de los tratamientos modernos, el suicidio como reacción al dolor y al mal estado general fue una complicación frecuente en los pacientes con R-2 (55). Nuestro paciente no tuvo complicaciones, 20 meses después de iniciado su eritema nodoso leproso y sin tratamiento específico en ese período. Predominó el cuadro clínico general, febril, adenopático y cutáneo, sin lesión neural ni ocular. No sabemos cuántos episodios reaccionales presentó en el transcurso de sus 20 meses de evolución de la enfermedad, sin diagnóstico $y$, por tanto, sin tratamiento adecuado (véase, evolución del paciente).

\section{Tratamiento}

El tratamiento de la reacción 2 debe tener en cuenta los siguientes aspectos:

1. Iniciar o no suspender la quimioterapia antibacilar con rifampicina, DDS y clofazimine, según el esquema del programa de control de la lepra (56).

2. Evaluar la magnitud de la reacción y considerar si el enfermo debe ser hospitalizado.

3. Controlar cuanto antes la neuritis aguda y las lesiones oculares, así como el edema de las manos y de los pies, cambios todos que determinan si la reacción es una urgencia; pueden conducir a incapacidades y lesiones irreversibles $(53,54)$.

4. Controlar el dolor, que ha sido causa de suicidio (55).

La reacción leve o moderada es manejable con aspirina, desde $500 \mathrm{mg}$ a $1 \mathrm{~g}$ cada ocho horas; la indometacina ( $50 \mathrm{mg}$ cada $8 \mathrm{~h}$ ) y la cloroquina (250 mg cada 8 h) también han sido útiles (38). La aspirina produce menos efectos colaterales; suprime la neuritis en una semana, mientras que la indometacina lo hace en dos (38).

En la reacción 2 persistente o crónica el clofazimine es útil, así: $100 \mathrm{mg}$, tres veces al día durante dos a tres meses y, luego, $100 \mathrm{mg} /$ día durante otro período semejante. El tratamiento total puede durar 8-12 meses, por lo cual se deben conocer sus efectos secundarios que incluyen la pigmentación parduzca cutánea, que tarda varios años en desaparecer, cólicos abdominales y diarreas, menos frecuentes mientras más baja sea la dosis diaria $(5,56,57)$. 
La talidomida se usa según la severidad de la reacción: 100 mg, cuatro veces al día o 200, dos veces al día, controlan la mayoría de las reacciones en 72 horas. Si el eritema nodoso leproso es crónico se usan 50-100 mg/día por varios meses (38). La talidomida se comenzó a usar en el tratamiento del ENL en 1965 (58), con muy buenos resultados. Ejerce su acción inhibiendo la síntesis de factor de necrosis tumoral alfa, promoviendo la degradación del mARN que lo codifica (59). También inhibe la quimiotaxis para los polimorfonucleares y su poder fagocítico; aumenta la producción de IL-4, pero tiene un efecto global estimulante de la funcion $\mathrm{TH}-1$, con aumento ligero en la producción de INFre IL-12 (60), por lo cual no es útil en la R-1. La talidomida es un teratógeno que produce alteración grave del desarrollo del esqueleto de los miembros superiores e inferiores, con focomelia $(61,62)$. En Brasil y Argentina se ha demostrado este efecto en más de 30 niños, hijos de madres con lepra que recibieron el medicamento para el eritema nodoso leproso (61), por lo cual se prohibió su uso en el Brasil. La talidomida es también un antiangiogénico importante, por lo cual su uso se ha extendido para el tratamiento de tumores como el sarcoma de Kaposi y el melanoma maligno, el carcinoma renal, el cáncer de la próstata, el mieloma múltiple y gliomas, con aprobación de la Food and Drug Administration de Estados Unidos en 1998 (63). Es un medicamento útil en el tratamiento del lupus eritematoso discoide resistente a la cloroquina (61). En Colombia se usa en varias entidades dermatológicas, como las aftas especialmente las que ocurren en pacientes con sida y en el prúrigo actínico (61). Que no se presente su efecto teratógeno es una responsabilidad directa del médico. Una sola pastilla de talidomida en los dos primeros meses del embarazo es suficiente para causar su efecto teratogénico (62). Otros efectos secundarios incluyen: neuropatía axonal sensitiva y motora, astenia, somnolencia, cefalea, náuseas, dolor abdominal, xerostomía, erupción cutánea, amenorrea, más notorios mientras más alta sea la dosis. Son poco frecuentes en lepra. La investigación reciente busca desarrollar análogos de la talidomida, que mantengan su efecto antifactor de necrosis tumoral alfa, suprimiendo la teratogenicidad (60).
Los corticoides están indicados en las reacciones graves y son mandatorios cuando hay neuritis manifiesta o silenciosa. La prednisona se usa en la dosis de 30-60 mg/día, con reducción semanal en $10 \mathrm{mg}$, según la respuesta clínica, hasta llegar

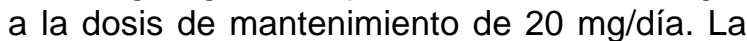
prednisolona se ha empleado a la dosis de 60$200 \mathrm{mg} /$ día, según la gravedad de la reacción. Debe bajarse la dosis hasta llegar a $30 \mathrm{mg} / \mathrm{día}$, evitando la dependencia del esteroide, frecuente en el eritema nodoso leproso crónico (38).

La pentoxifilina ha dado resultados buenos a la dosis de $400 \mathrm{mg}$ cada $8 \mathrm{~h}$, junto con $100 \mathrm{mg}$, cada $8 \mathrm{~h}$ de clofazimine $(64,65)$, mientras que para otros ha sido de poca utilidad (66). No se ha utilizado entre nosotros.

Algunas reacciones 2 son crónicas y difíciles de manejar. La combinación de clofazimine, talidomida y esteroides puede usarse, disminuyendo la dosis de esteroides y teniendo en cuenta que es esencial evitar el daño neural y ocular. En toda reacción 2 deben buscarse factores coadyuvantes secundarios como focos sépticos y tiñas.

Los síntomas oculares deben ser manejados por el oftalmólogo. La iritis requiere el uso de atropina al $1 \%$ y de cortisona al $1 \%$, al menos, 3 veces al día (38).

En el embarazo se pueden usar los mismos esquemas terapéuticos, excepto la talidomida, que tiene contraindicación absoluta.

En algunos casos, la descompresión quirúrgica del tronco nervioso afectado, con trasposición del mismo, ha sido de utilidad con beneficio del paciente (38).

En conclusión, las reacciones en lepra son eventos comunes en los enfermos colombianos, con mayor frecuencia, la reacción tipo 2 . Su presentación clínica es variada, con síntomas agudos importantes; junto con los nódulos cutáneos, las adenopatías y las neuritis permiten sospecharla y diagnosticarla, en el laboratorio se destaca la leucocitosis con neutrofilia y la albuminuria.

La R-2 es una urgencia médica potencial, capaz de originar daño neural, ocular, testicular y 
discapacidad grave de las manos y de los pies. Su manejo médico y fisioterapéutico permite evitar las incapacidades que produce. Es un tema en el que debe tener conocimiento preciso todo el personal de salud, principalmente el encargado de la salud pública y de la prevención de discapacidades.

\section{Agradecimientos}

A Jaime Cortés del Hospital Regional San Salvador de Chiquinquirá, Boyacá, por su ayuda en la recopilación de los datos clínicos y en la toma de las fotografías clínicas del paciente.

\section{Referencias}

1. Rodríguez G, Orozco L, editores. Lepra. Bogotá: Instituto Nacional de Salud; 1996.

2. Jopling WJ. Handbook of leprosy. Third edition. London: W. Heinemann Medical Books; 1984.

3. Sehgal VN. Reactions in leprosy. Clinical aspects. Int $\mathrm{J}$ Dermatol 1987;26:278-85.

4. Job CK, Gude S, Macaden VP. Erythema nodosum leprosum. A clinico-pathologic study. Int J Lepros 1964; 32:177-84.

5. Jolliffe DS. Leprosy reactional states and their treatment. Br J Dermatol 1977;97:345-52.

6. Gottlieb LS, Southgate MT. Acute adenopathy in a young man. JAMA 1973;224:1737-46.

7. Scully RE, Marv EJ, McNelly BU. Case records of the Massachusetts General Hospital. Case 49-1985. N Eng J Med 1985;313:1164-72.

8. Albert DA, Weisman MH, Kaplan R. The rheumatic manifestations of leprosy (Hansen's disease). Medicine 1980;59:442-8.

9. Editorial. Rheumatic manifestation in leprosy. Lancet 1981;1:648-9.

10. Rodríguez G, Berrío J, Sarmiento L. La lepra y el riñón. Biomédica 1999;19:45-55.

11. Karat ABA, Karat S, Job CK, Sudarsanam D. Necrotizing lepromatous lymphadenitis: an erythema-nodosumleprosum-like reaction in lymph nodes. Br Med J 1968; 4:223-4.

12. Fiallo P, Pesce C, Lenti E, Nunzi E. Short report: erythema nodosum leprosum lymphadenitis. Am J Trop Med Hyg 1995;52:297-8.

13. Rea TH, Levan NE. Erythema nodosum leprosum in a general hospital. Arch Dermatol 1975;111:1575-82.

14. Manandhar R, LeMaster JW, Roche PW. Risk factors for erythema nodosum leprosum. Int $\mathrm{J}$ Lepr Other Mycobact Dis 1999;67:270-8.
15. Kar HK, Mohanty HC, Mohanty GN, Nayak UP. Clinicopathological study of lymph node involvement in leprosy. Lepros India 1983;55:725-42.

16. Ioachim HL. Mycobacterium leprae lymphadenitis. En: Lymph node pathology. Second edition. Philadelphia: JB Lippincott Co.; 1994. p.130-4.

17. García NM. Valor diagnóstico de la punción ganglionar en la lepra. Rev Higiene 1938;19:5-45.

18. Sharma A, Sharma VK, Rajwanshi A, Das A, Kaur I, Kumar B. Presence of $M$. leprae in tissues in slit skin smear negative multibacillary (MB) patients after WHOMBR. Lepr Rev 1999;70:281-6.

19. Bhargara P, Kuldeep CM, Mathur KN. Erythema dolosum leprosum in subgroups of lepromatous leprosy. Lepr Rev 1997;68:373-85.

20. Hussain R, Lucas SB, Kifayet A, Jamil S, Raynes J, Ugaili $Z$ et al. Clinical and histological discrepancies in diagnosis of ENL reactions classified by assessment of acute phase proteins SAA and CRP. Int J Lepros Other Mycobact Dis 1995;63:222-30.

21. Harada K. Periodic acid-methenamine silver stain for mycobacteria in tissue sections. Stain Tecnol 1976;51: 278-80.

22. Naranjo P, Rodríguez G, Rodríguez J. La coloración de Ziehl-Neelsen en histopatología. Biomédica 1988;8: 84-90.

23. Singh S, Jenner PJ, Shanker NP, Ramu G, Colston MJ, Prasad HK, et al. Critical residues of the Mycobacterium leprae LSR recombinant protein discriminate clinical activity in erythema nodosum leprosum reactions. Infec Inmun 1994;62:5702-5.

24. Modlin RL, Mehra V, Jordan R, Bloom BR, Rea TH. In situ and in vitro characterization of the cellular immune response in erythema nodosum leprosum. J Immunol 1986;136:883-6.

25. Modlin RL, Gebhard JF, Taylor CR, Rea TH. In situ characterization of $T$ lymphocyte subsets in the reactional states of leprosy. Clin Exp Immunol 1983;53: 17-24.

26. Sreenivasan P, Misra RS, Wilfred D, Nath I. Lepromatous leprosy patients show $T$ helper 1 -like cytokine profile with differential expression of interleukin-10 during type 1 and 2 reactions. Immunology 1998;95: 529-36.

27. Nath I, Vemuri N, Reddi AL, Bharadwaj M, Brooks $\mathbf{P}$, Colston MJ, et al. Disregulation of IL-4 expression in lepromatous leprosy patients with and without erythema nodosum leprosum. Lepr Rev 2000;71 (Suppl.):S130-7.

28. Nath I, Vemuri N, Reddi AL, Jain S, Brooks P, Colston MJ, et al. The effect of antigen presenting cells on the cytokine profiles of stable and reactional lepromatous leprosy patients. Immunol Lett 2000;75:69-76. 
29. Moraes MO, Sarno EN, Almeida AS, Saraiva BC, Nery JA, Martins RC, et al. Cytokine mRNA expresión in leprosy: a possible role for interferon gamma and interleukin 12 in reactions (RR and ENL). Scand $J$ Immunol 1999;50:541-9.

30. Moraes MO, Sarno EN, Teles RM, Almeida AS, Saraiva BC, Nery JA, Sampaio EP. Anti-inflammatory drugs block cytokine mRNA accumulation in the skin and improve the clinical condition of reactional leprosy patients. J Invest Dermatol 2000;115:935-41.

31. Schon T, Leekassa R, Gebre N, Sundqvist T, Bizuneh E, Britton S. High dose prednisolone treatment of leprosy patients undergoing reactions is associated with a rapid decrease in urinary nitric oxide metabolites and clinical improvement. Lepr Rev 2000;71:355-62.

32. Modlin RL, Bloom BR. Immune regulation: learning from leprosy. Hosp Pract 1993;28:71-84.

33. Vieira LM, Sampaio EP, Nery JA, Duppre NC, Alburquerque EC, Schelinberg MA et al. Inmunological status of ENL (erythema nodosum leprosum) patients: its relationship to bacterial load and levels of circulating IL-2R. Rev Inst Med Trop Sao Paulo 1996,38:103-11.

34. Wemambu SNC, Turk JL, Waters MFR, Rees RJW. Erythema nodosum leprosum: a clinical manifestation of the Arthus phenomenon. Lancet 1969;ii:933-5.

35. Moran CJ, Turk JL, Ryder G, Waters MFR. Evidence for circulating immune complexs in lepromatous leprosy. Lancet 1972;ii:572-3.

36. Rojas-Espinosa O, Mendez-Navarrette I, EstradaParra S. Presence of Clq-reactive immune complexes in patients with leprosy. Clin Exp Immunol 1972;12: 21523.

37. Saunderson $\mathbf{P}$, Gebre $\mathbf{S}$, Byass $\mathbf{P}$. ENL reactions in the multibacillary cases of the AMFES cohort in central Ethiopia: incidence and risk factors. Lepr Rev 2000;71: 318-24.

38. Lockwood DNJ. The management of erythema nodosum leprosum: current and future options. Lepr Rev 1996;67:253-9.

39. Girdhar BK, Girdhar A, Kumar A. Relapses in multibacillary leprosy patients: effect of length of therapy. Lepr Rev 2000;71:144-53.

40. Duncan ME, Pearson JM. The association of pregnancy and leprosy. III. Erythema nodosum leprosum in pregnancy and lactation. Lepr Rev 1986;59:129-36.

41. Greenfield S, Jordan C. The clinical investigation of lymphadenopathy in primary care practice. JAMA 1978; 240:1388-93.

42. Selavasekar A, Ebenazar GJ, Perthecbarajan M. Lepromatous lymphadenopathy and concomitant tuberculosis axillary lymphadenitis with sinus. A case report. Lepr Rev 1999;70:345-50.
43. Kolker SE, Manz HJ, Schwartz DA. Syphilis. En: Connor DH, Chandler FW et al., editors. Pathology of infectious diseases. First edition. Stanford, Connecticut: Appleton \& Lang; 1997. p.833.

44. Rodríguez G, Torres B, Motta A. Angiomatosis bacilar. Biomédica 2002;22:141-8.

45. LeBoit PE. Bacillary angiomatosis. En: Connor DH, Chandler FW et al., editors. Pathology of infectious diseases. First edition. Stanford, Connecticut: Appleton \& Lange; 1997. p.407-20.

46. Rodríguez G. Leishmaniasis y sistema linfático. Acta Med Colomb 2000;25:199-203.

47. Barral A, Barral-Netto M, Almeida R, De Jesús A, Grimaldi JR Q, Netto EM et al. Lymphadenopathy associated with Leishmania braziliensis cutaneous infection. Am J Trop Med Hyg 1992;47:587-92.

48. Barral A, Guerreiro J, Bomnfim G, Correia D, BarralNeto M, Carvalho EM. Lymphadenopathy as the first sign of human cutaneous infection by Leishmania braziliensis. Am J Trop Med Hyg 1995;53:256-9.

49. Miettinen M. Histological differencial diagnosis between lymph node toxoplasmosis and other bening lymph node hyperplasias. Histopathology 1981;5:205-16.

50. Aisner SC, Aisner J, Moravec C. Acquired toxoplasmic limphadenitis with demostration of cyst form. Am J Clin Path 1983;70:125-7.

51. Rodríguez G, Motta A, Ordóñez N. Estudio histopatológico de 19 biopsias cutáneas de pacientes con sida e histoplasmosis diseminada. Biomédica 2001; 21:107-15

52. Siegel RJ. Human immunodeficiency virus-associated lymphoid disease. En: Connor DH, Chandler FE et al, editors. Pathology of infectious diseases. Stanford, Conn.: Appleton \& Lange; 1997. p.161.

53. Rodríguez G. Reacciones en lepra. Urgencias en Dermatología. Bogotá: Plough Farmacéutica; 1999. p.181-9.

54. Yoder LJ. Management of reactions in Hansen's disease. Star 1987;46:1-7.

55. Bjune G. Reactions in leprosy. Lepr Rev 1983;56:61S$67 \mathrm{~S}$.

56. Ministerio de Salud. Guía de atención de la lepra. En: Normas técnicas y guías de atención. Bogotá: Ministerio de Salud; 2000.

57. Millan J, Bobin P. Traitement des reactions et des névrites. En: Sansarricq $\mathrm{H}$, editor. La lepre. Paris: Ellipses; 1995. p.250.

58. Sheskin J. Thalidomide in the treatment of lepra reactions. Clin Pharmacol Therap 1965;6:303-14.

59. Moreira AL, Sampaio EP, Zmuidzinas A, Frindt $\mathbf{P}$, Smith KA, Kaplan G. Thalidomide exerts its inhibitory 
action on tumor necrosis factor alfa by enhancing mRNA degradation. J Exp Med 1993;177:1675-80.

60. Kaplan G. Potential of thalidomide and thalidomide analogues as immunomodulatory drugs in leprosy and leprosy reactions. Lepr Rev 2000;71:S117-S120.

61. Rodríguez RA. La otra cara de la talidomida. Biomédica 1995; 15:89-92.

62. Castilla EE, Ashton-Prolla P, Barrera-Mejía E, Brunoni D, Cavalcanti DP, Correa-Neto J, et al. Thalidomide, a current teratogen in South America. Teratology 1996;54:273-7.

63. Richardson P, Hideshima P, Anderson K. Thalidomide: emergency role in cancer medicine. Ann Rev Med 2002; 53:629-57.
64. Gómez M, Macías C, Welsh O. New therapeutic approach to type II leprosy reaction. En: Clinical Dermatology 2000. An International Congress. Vancouver 2831 May, 1996.

65. Nery JA, Perisse Ar, Sales AM, Vieira LM, Souza RV, Sampaio EP, et al. The use of pentoxyfilline in the treatment of type 2 reactional episodes in leprosy. Indian J Lepr 2000;72:457-67.

66. Rea TH. Clinical and immunological perspectives on the reactional states of leprosy. En: Clinical Dermatology 2000. An International Congress, Vancouver, 28-31 May 1996. 